\title{
Optical non-invasive diagnostics of microcirculatory-tissue systems of the human body: questions of metrological and instrumentation provision
}

\author{
Evgeny A. Zherebtsov, Victor V. Dremin, Angelina I. Zherebtsova, Irina N. Makovik, \\ and Andrey V. Dunaev* \\ Orel State University named after I.S. Turgenev, 95 Komsomolskaya St., Orel 302026, Russia
}

*e-mail: dunaev@bmecenter.ru

\begin{abstract}
Optical techniques are one of the most promising non-invasive technologies for the diagnosis of medical conditions. This work is devoted to the current state of the optical non-invasive diagnostics (OND), especially about instrumentation as well as methodological and metrological provision for this technology. In some details Laser Doppler Flowmetry (LDF) and Fluorescence Spectroscopy (FS) diagnostics methods are considered. The main conclusion is that it is necessary to solve the problems of metrological support OND devices of these types. It is also important to develop a methodology based on diagnostic criteria obtained from a relationship between a number of OND methods and others. (C) 2016 Journal of Biomedical Photonics \& Engineering.
\end{abstract}

Keywords: optical non-invasive diagnostics, blood microcirculation, biotissue, laser Doppler flowmetry, fluorescence spectroscopy.

Paper \#3129 received 2016.11.28; accepted for publication 2016.12.29; published online 2016.12.31. doi: 10.18287/JBPE16.02.040305. [Saratov Fall Meeting 2016 Special Issue].

\section{References}

1. V. V. Tuchin (ed.), Handbook on Optical Biomedical Diagnostics, SPIE Press, Bellingham (2002).

2. G. E. Nilsson, T. Tenland, and P. A. Oberg, "Evaluation of a laser Doppler flowmeter for measurement of tissue blood flow," IEEE Trans Biomed Eng BME 27(10), 597-604 (1980).

3. A. N. Obeid, N. J. Barnett, G. Dougherty, and G. Ward, "A critical-review of laser Doppler flowmetry," Journal of Medical Engineering \& Technology 14(5), 178-181 (1990).

4. M. J. Leahy, F. F. M. de Mul, G. E. Nilsson, and R. Maniewski, "Principles and practice of the laser-Doppler perfusion technique," Technol Health Care 7(2-3), 143-162 (1999).

5. A. I. Krupatkin, and V. V. Sidorov, Laser Doppler flowmetry of Blood Microcirculation, Meditcina-Press, Moscow (2005).

6. M. Bracic, and A. Stefanovska, "Wavelet-based analysis of human blood-flow dynamics," Bulletin of Mathematical Biology 60(5), 919-935 (1998).

7. A. V. Tankanag, and N. K. Chemeris, "Application of the adaptive wavelet transform for analysis of blood flow oscillations in the human skin,” Phys. Med. Biol. 53(21), 5967-5976 (2008).

8. J. R. Lakowicz (ed.), Principles of Fluorescence Spectroscopy, Kluwer Academic Publishers, $3^{\text {rd }}$ ed., Springer, New York (2006). ISBN: 10 0-387-32278-1.

9. M. A. Mycek, and B. W. Pogue (eds.), Handbook of Biomedical Fluorescence, CRC Press, Taylor \& Francis (2003). ISBN 978-0-824-70955-6.

10. D. Roblyer, R. A. Schwarz, and R. R. Richards-Kortum, "Fluorescence Spectroscopy," in Handbook of Biomedical Optics, D. A. Boas, C. Pitris, and N. Ramanujam, (eds.), CRC Press, Taylor \& Francis, 217-232, (2011).

11. A. V. Dunaev, E. A. Zherebtsov, D. A. Rogatkin, N. A. Stewart, S. G. Sokolovski, and E. U. Rafailov, "Substantiation of medical and technical requirements for noninvasive spectrophotometric diagnostic devices," Journal of Biomedical Optics 18(10), 107009 (2013). 
12. A. V. Dunaev, V. V. Dremin, E. A. Zherebtsov, I. E. Rafailov, K. S. Litvinova, S. G. Palmer, N. A. Stewart, S. G. Sokolovski, and E. U. Rafailov, "Individual variability analysis of fluorescence parameters measured in skin with different levels of nutritive blood flow," Medical Engineering and Physics 37(6), 574-583 (2015).

13. S. L. Jacques, “Optical properties of biological tissues: a review,” Phys. Med. Biol. 58(11), R37-R61 (2013).

14. V. V. Dremin, and A. V. Dunaev, "How the melanin concentration in the skin affects the fluorescencespectroscopy signal formation," Journal of Optical Technology 83(1), 43-48 (2016).

15. I. E. Rafailov, V. V. Dremin, K. S. Litvinova, A. V. Dunaev, S. G. Sokolovski, and E. U. Rafailov, "Computational model of bladder tissue based on its measured optical properties," Journal of Biomedical Optics 21(2), 025006 (2016).

16. I. Rafailov, S. Palmer, K. Litvinova, V. Dremin, A. Dunaev, and G. Nabi, "A novel excitation-emission wavelength model to facilitate the diagnosis of urinary bladder diseases," Proc. SPIE 9303, 93030W (2015).

17. V. V. Dremin, E. A. Zherebtsov, I. E. Rafailov, A. Y. Vinokurov, I. N. Novikova, A. I. Zherebtsova, K. S. Litvinova, A. V. Dunaev, "The development of attenuation compensation models of fluorescence spectroscopy signals," Proc. SPIE 9917, 99170Y (2016).

18. T. Binzoni, T. S. Leung, M. L. Seghier, and D. T. Delpy, “Translational and Brownian motion in laser-Doppler flowmetry of large tissue volumes," Physics in Medicine and Biology 49(24), 5445-5458 (2004).

19. I. Fredriksson, M. Larsson, F. Salomonsson, and T. Strömberg, "Improved calibration procedure for laser Doppler perfusion monitors," Proc. SPIE 7906, 790602 (2011).

20. A. Liebert, M. Leahy, and R. Maniewski, "A calibration standard for laser-Doppler perfusion measurements," Review of Scientific Instruments 66(11), 5169-5173 (1995).

21. M. Larsson, W. Steenbergen, and T. Stromberg, "Influence of optical properties and fiber separation on laser Doppler flowmetry," Journal of Biomedical Optics 7(2), 236-243 (2002).

22. E. A. Zherebtsov, A. I. Zherebtsova, A. V. Dunaev, and K. V. Podmaster'ev, "Method and device for metrological control of laser Doppler flowmetry devices," Biomedical Engineering 48(4), 191-195 (2014).

23. A. V. Dunaev, E. A. Zherebtsov, D. A. Rogatkin, N. A. Stewart, S. G. Sokolovski, and E. U. Rafailov, "Novel measure for the calibration of laser Doppler flowmetry devices," Proc. SPIE 8936, 89360D (2014).

24. V. V. Dremin, V. V. Sidorov, A. I. Krupatkin, G. R. Galstyan, I. N. Novikova, A. I. Zherebtsova, E. A. Zherebtsov, A. V. Dunaev, Z. N. Abdulvapova, K. S. Litvinova, I. E. Rafailov, S. G. Sokolovski, and E. U. Rafailov, "The blood perfusion and NADH/FAD content combined analysis in patients with diabetes foot," Proc. SPIE 9698, 969810 (2016).

\section{Introduction}

At present, optical non-invasive technologies [1], such as laser Doppler flowmetry (LDF) and fluorescence spectroscopy (FS) are widely utilized for the diagnosis of peripheral tissues, the assessment of blood microcirculation and biological tissue concentrations of enzymes. However, these technologies have a number of general problems unsolved today. In particular, the problems are low reliability and methodological limitations. This leads these methods having no wide and proper application in clinical practice. Thus, the solution to these problems of OND is to increase their efficiency and applicability in clinical practice. Possible ways to resolve the task can be improvement of the metrological assurance as well as further development of the instrumental tools. For example, the highperformance applications in clinical practice will allow us to identify the disease at level of tissue systems in the early stages of development. Undoubtedly it will reveal new horizons in the medicine of occupational diseases as well as reduce the risks of the socially significant diseases in the social and active segment of the population that will have a positive impact on the structure of the market of labour and the economy as a whole.

It is common knowledge that the LDF method is widely used in the research programs of various research teams and allows to estimate the intensity of peripheral blood flow in the microvasculature. The unit of measurement of the method in most cases is the index of microcirculation $\left(I_{m}\right)$. The value of the index by convention is expressed in arbitrary perfusion units and is proportional to the average concentration of red blood cells (RBC) as well as their average velocity. Practically, the $I_{m}$ is the result of processing of the signal from the photodetector, which is formed in photomixing of signals from the reference and frequency-shifts (in the band from $1 \mathrm{~Hz}$ to $24 \mathrm{kHz}$ ) due to the Doppler effect during scattering on RBC coherent light fluxes [2-4]. The time domain recorded signal of $I_{m}$ consists of variable and constant components. The constant component - this is the mean perfusion of blood over a selected time interval. The variable component of the signal reflects the rhythms of frequency regulation of blood flow (fluxomotions) which appear due to the regulation of blood circulation and other physiological factors. Both components provide important information for diagnosing a number of diseases [5].

One of the weaknesses of the LDF method is its insufficient metrological support, leading to a lack of representation in medical practice. There is still no accurate, convenient and inexpensive tool (test object, optical phantom) to monitor the technical condition of LDF devices. Therefore, for the further development and wider application of the method in practice, it is vital to develop the system that can quantitatively 
reproduce the reference and Doppler-shifted light fluxes in the correct proportions. The estimated test facility should provide an opportunity to quickly and comprehensively monitor the technical condition of both optical and electronic parts of the measurement channel. In particular, the system should reproduce the variable and constant components of the perfusion signal. It becomes important for the diagnosis of some diseases, that there is a need for accurate evaluation of fluctuations in vascular blood [6-7]. Thus, the level of the reproducible signal should be accurate and be able to vary in some range. Another important requirement is the versatility and ease of use.

Fluorescent spectroscopy (FS) is becoming more widely used in chemistry, biology, in various fields of medical technology and medicine in general. These methods are highly sensitive and provide a unique opportunity to study the excited states of molecules, photochemical reactions, dynamics of fast molecular processes, structures, and properties of complex biochemical and cellular systems. FS provides effective and non-invasive optical diagnostics, primarily in medical areas such as oncology, transplantation, cosmetology and surgery. The FS method is based on exciting fluorescence from tissue endogenous and exogenous fluorophores and recording the emission in the visible spectral region [8-9]. FS is a good method to differentiate benign and malignant tumours of various origins [10]. FS is also used as a tool to monitor the dynamics of the processes occurring in the tissues, during cancer treatments such as radiotherapy. Many purulent wounds, burns and other destructive inflammatory processes are accompanied by changes in the fluorescent activity of the tissues, which occurs due to a misbalance in accumulation of natural fluorophores: FAD, NADH, lipofuscin, porphyrins, structural proteins, etc.

However, fluorescence spectroscopy of biological tissue is a complicated technique that depends on the temperature, topological heterogeneity, different properties of each sample, etc. Therefore, the reliability of FS is affected by multiple factors, including the availability of data concerning the scattering and absorbing properties of specific tissues in specific conditions, light pollution at the optical fibre tip and instrument errors such as excitation source instability, photo-detector limitations, light filter precision, grating precision, CCD performance, etc. This study aims to fill some gaps in the FS method.

The study conducted by the authors represents an attempt at forming a comprehensive, systematic and unifying approach of solving the above mentioned problems of the diagnostic methods. The problems of accuracy, variation and reproducibility of measurements are investigated. The obtained results can improve the reliability of clinical diagnostic results by the methods of FS and LDF. To assess the variability of the diagnostic parameters obtained by FS and LDF methods appropriate experimental studies have been conducted. As main equipment, the multifunction laser diagnostic system "LAKK-M" (SPE "LAZMA" Ltd., Russia) was chosen. The experiments were conducted with the participation of healthy volunteers. Comparative analysis of the collected data showed that the relative variation of the parameters for all volunteers has the quite homogeneous mean level and can range from 10 to $40 \%$ depending on the determined parameter. It has been found that total scatter and, consequently, the final diagnostic result can be affected by several factors. Thus, the blood supply of the biological tissue affects both attenuation of the intensity of the exciting radiation and the excited fluorescence radiation $[11,12]$.

Also, the effect of melanin on the fluorescence signal was investigated. This skin component is one of the most important natural pigments and has a significant impact on the optical properties of the skin. Influence of melanin was assessed in different ethnic skin types, including European, Indian, Arabic and African [13]. It is found that with increasing the melanin concentration the fluorescence signal decays monotonically. For the tasks of medical instrument engineering recommendations about the influence of the effect on signal-to-noise ratio of the photodetector were given $[12,14]$.

In order to describe and predict the effects of the above factors on the obtained FS spectrum, a Monte Carlo model of propagation of optical radiation in epithelial biological tissues was developed [12, 14-17]. Such an approach to solve this problem seems to be prospective and shows good accuracy of the modelling results.

\section{New approaches of metrological support of devices for laser Doppler flowmetry}

At present, as a test objects for the LDF measurements, colloidal solutions of light-scattering particles are most widely used. The tissue phantoms simulate the movement of RBC by Brownian motion of the microparticles of certain size and concentration. The speed of Brownian motion is calculated from the theoretical dependences on temperature as well as particle shape parameters [18-20]. An example of such calibration system is the test object "Motility Standard" (Perimed AB, Sweden) [21]. However, the test object is not stable in time and undergoes desiccation. In addition, the test object can reproduce only one level of the perfusion.

Conducted experimental research of the colloid solution standard together with LDF-channel (single mode laser, wavelength $1064 \mathrm{~nm}$ ) of "LAKK-M" system demonstrated significant methodological shortcomings of this approach. The results of periodic monitoring of the reproduced level of LDF signal for channel "LAKK-M" by "Motility Standard" during 8 months is presented at Figure 1.

The fibre optic probe of the reference LDF channel was submerged in the solution and the reproduced signal was recorded for 30-minute. One possible reason for obtaining a sufficiently large spread of results is that the solution has changed their characteristics over time (its shelf life is no more than six months). Also, significant drawback of this approach of calibration is the enormous sensitivity to any vibration of the surface table. To overcome the described problems new 
approach for monitoring the technical condition of the LDF instruments was proposed.

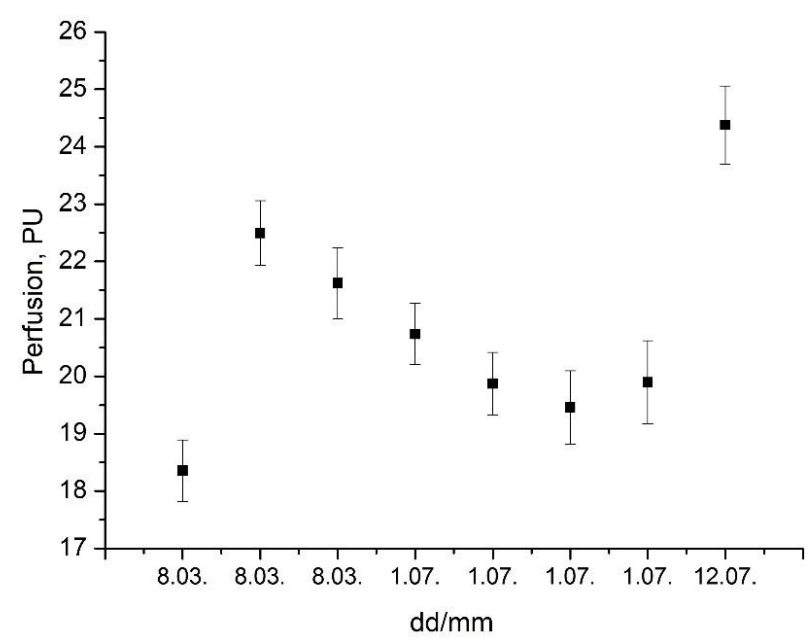

Fig. 1 The results of periodic monitoring of the reproduced level of LDF signal by "Motility Standard" (Perimed AB, Sweden).

The approach is based on the use of a new test object. General view of the setup is presented at Figure $2 \mathrm{a}$. In the setup quantitatively reproducing of the reference and Doppler-shifted light fluxes is provided by the scattering on oscillating standard Lambertian reflector covered by immovable semi-transparent glass plate. The main basis of operation is presented at Figure $2 b$. The electromechanical transducer 3 converts electrical signal into mechanical vibrations in the moving diffuse reflector 2 . In order to create a constant component of the signal, laser radiation from the probe fiber (aperture of source $S_{i n}$ ) passes first through a fixed semi-transparent plane-parallel plate 1 . The reflected back from the plate radiation does not undergo Doppler shift. Laser radiation is scattered by oscillating diffusing Lambertian surface and receives the component of the Doppler shift. Both radiation fluxes are collected by receiving fiber with aperture $S_{\text {out }}$ and processed by the checked LDF device. The reflector 2 is driven by highprecision piezo actuator with the oscillation frequency of 0 to $100 \mathrm{~Hz}$. In that way, this test facility realized the possibility of reproducing a constant level of perfusion as well as blood filling fluctuations (fluxmotions) in the microvasculature in the frequency range of 0.01 to $2 \mathrm{~Hz}$. By varying modes of operation, it is possible to simulate a variety of patterns of LDF signal. Thus, the approach provides the tool of checking during one procedure both optical and electronic parts of the measurement channel. This test facility allows to make a technical check of the device as well as metrological control of its condition $[22,23]$. No doubt further improvement of the metrological provision of LDF method will rise this diagnostic technology to a new level of effectiveness and reliability.

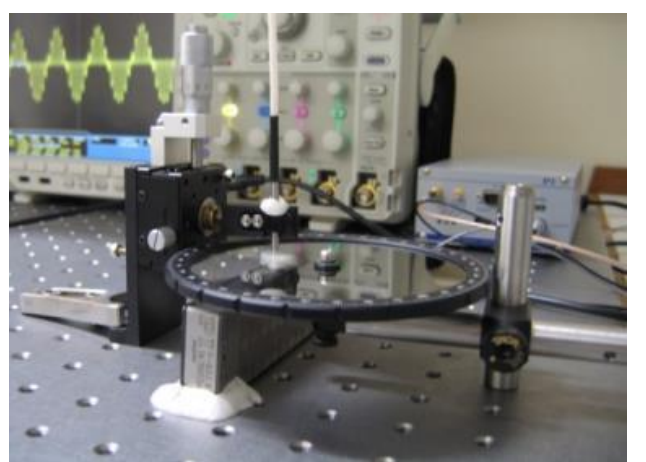

(a)

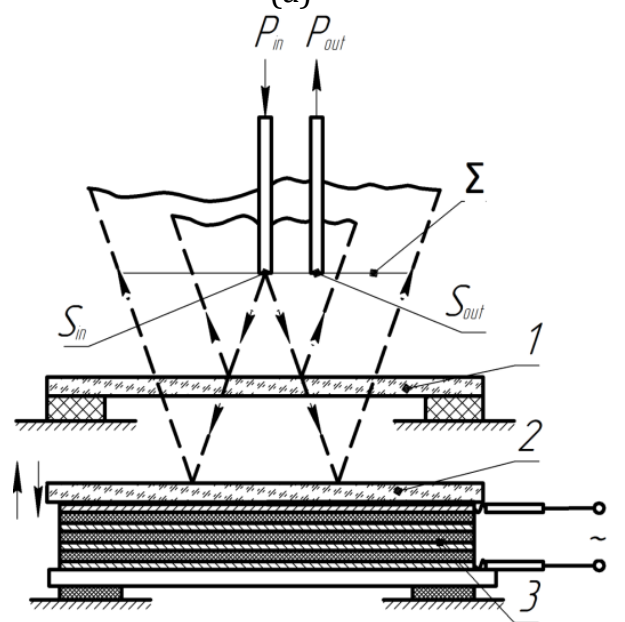

(b)

Fig. 2 The experimental setup for metrological check of LDF devices based on the oscillating standard Lambertian reflector: (a) general view; (b) the main basis of operation:

1 - semi-transparent plane-parallel plate, 2 - moving diffuse reflector, 3 - electromechanical transducer (batch piezoelectric actuator), $S_{i n}$ - aperture of source, $S_{\text {out }}$ - aperture of the receiver, $\Sigma$ - plane coincidence of aperture of receiver and source.

\section{Problem with methodological and instrumentation provision of fluorescence spectroscopy for medicine}

Experiments were conducted to assess the performance of 2 identical FS-channels. Measurements were conducted on a solution of porphyrin in PBS solution. The cuvette with the biomarker was placed on a white PTFE reflection standard. A concentration of $0.25 \mathrm{mM}$ was used. The depth of immersion of the fibres in the cuvette was about $5 \mathrm{~mm}$. Figure 3 shows some porphyrin fluorescence spectra (Figure $3 \mathrm{a}$ and $3 \mathrm{~b}$ for green $(532 \mathrm{~nm})$ and red $(635 \mathrm{~nm})$ fluorescence excitation lines respectively). 

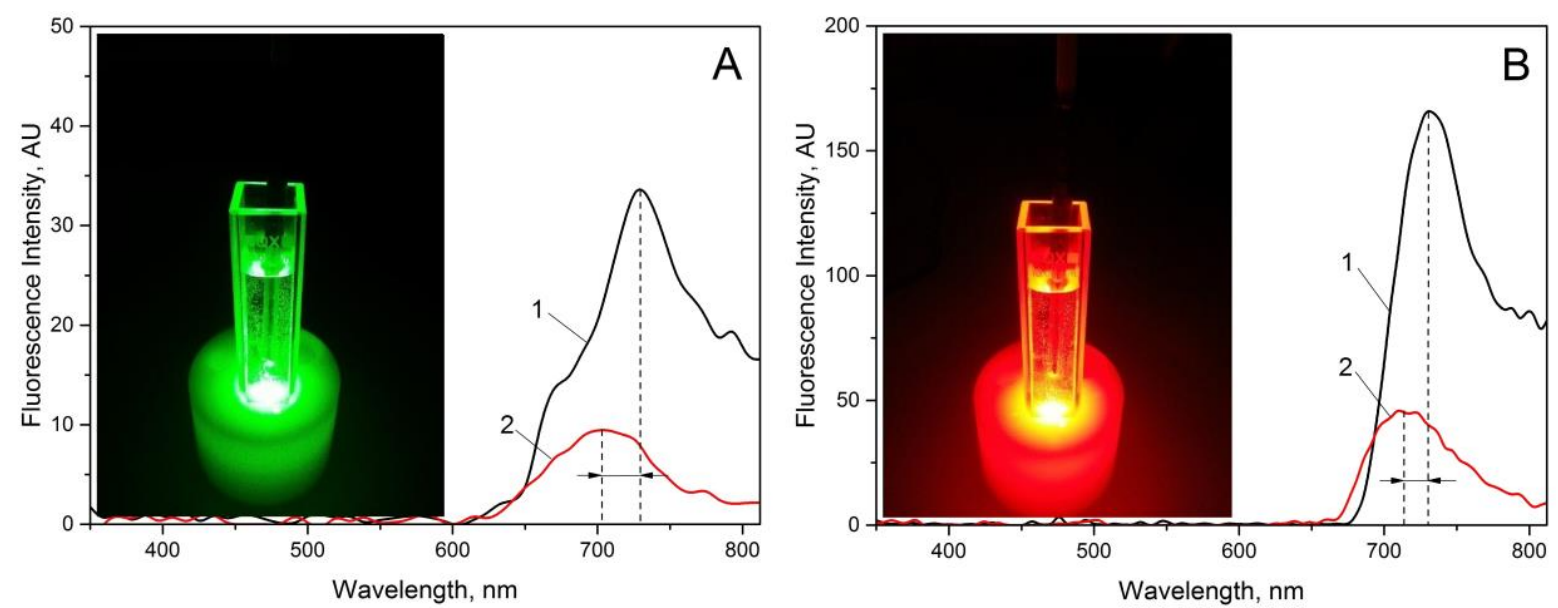

1 - Device №1; 2 - Device №2

Fig. 3 Examples of fluorescence spectra: for porphyrin on the green (a) and red (b) lines excitation.
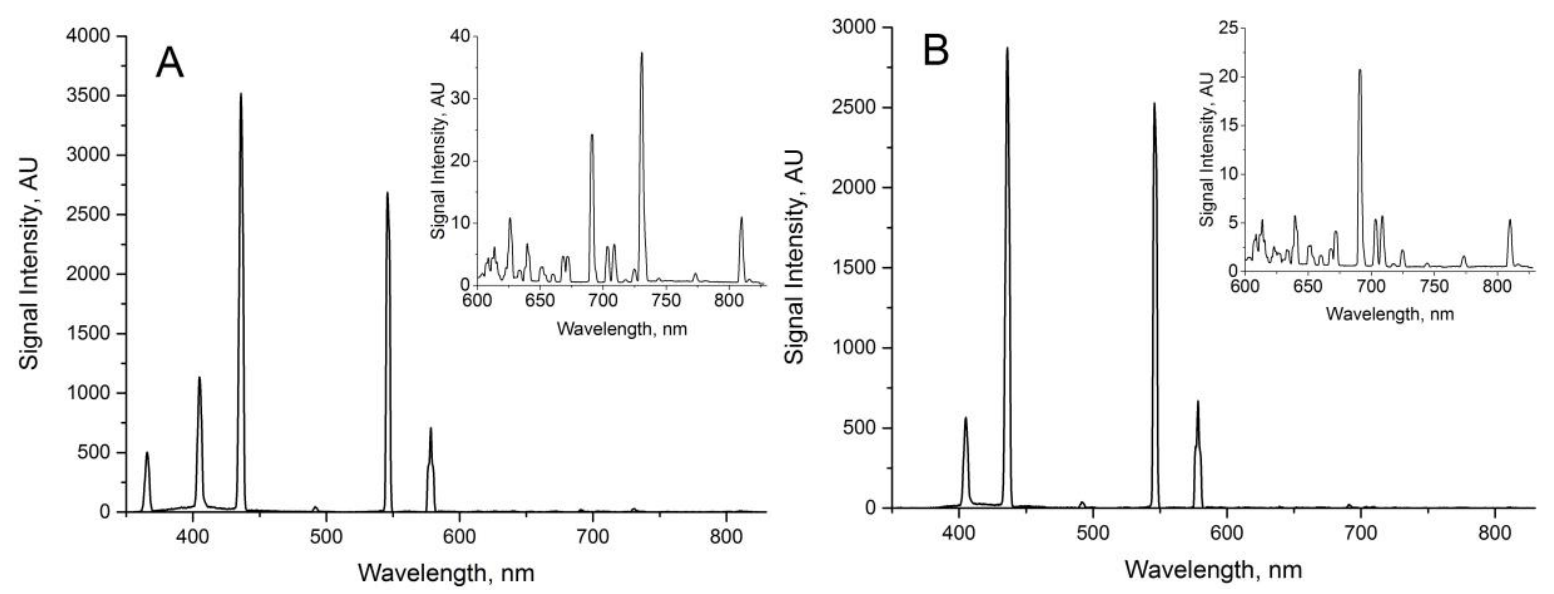

Fig. 4 The registered spectrum of mercury lamp without a filter (a) and with UV filter (b).

In addition to physical errors, there were also a number of technical errors in the method. Thus, even the minor parameter changes in optical radiation of the device, differences between separate receiver sites and individual nodes of the optical system, may visibly influence the shape of the spectral curve and, consequently, the final diagnostic result when measuring the fluorescent spectra. In the figures, dotted lines represent the peaks of fluorescent biomarkers.

As seen in Figure 3, for the two compared FS channels fluorescence intensity values differ greatly in amplitude. The reason for this may be a different spectral sensitivity of the transfer function and resolution of different diagnostic instruments, since cell CCD arrays have unequal sensitivity, i.e. even if the signal is homogeneous, illumination produced is different. The magnitude of this heterogeneity is typically less than $5 \%$. However, this does not eliminate the problem of reducing CCD noise itself, as fluorescent applications often work with the signals in the tens of photons per cell. For this reason, in systems which require high photometric accuracy such as this, it is necessary to apply the same non-uniformity correction algorithms as well as adjusting dark current. Spectral characteristics of the CCD, however small, affect the quality of the signal. Often, the output electronic cascades are an even more intense source of noise. In this regard, attention should be paid to the rational matching of a receiver and a follow-up system.

As mentioned above, the different nodes of the optoelectronic system appear to be the primary sources of noise, interference and other distortion. Thus, a set of interchangeable filters is installed on the input of the devices polychromator, damping the radiation at given source wavelengths to required levels. As studies have shown with use of a mercury gauge lamp (Fig.4), utilised filters affect the sensitivity of the measurement channel. More specifically, they weaken not only the backscattered radiation, but the fluorescence emission of various fluorophores, which affects the whole recorded spectra and calculated parameters.

One may infer the need for more precise filters with a narrow band filter and a high percentage transmittance of other wavelengths. Based on the received results, we can conclude on the need to develop methods of metrological monitoring for FS devices. Metrological provision should cover both the spectral calibration of the instrument readings and the normalisation of quantitative fluorescence, based on modelling the endogenous fluorescence of the investigated fluorophores. Creation of new FS hardware also requires new approaches to software development for 
the processing and analysis of diagnostic information to develop practical methods for its application in applied clinical medicine. It should be emphasized that addressing the issues of metrological support for the FS method can bring this diagnostic technology to a new level.

\section{Conclusion}

The presented results demonstrate the relevance of instrumentation, methodological and metrological provision for these technologies in general and especially for the medical application of the LDF- and FS-devices and combined systems. The combined use of OND technologies with functional (provocative) tests allows increasing the repeatability of results and accuracy of diagnostics (for example, LDF and FS methods allows to predict the development of trophic disorders and the diabetic foot syndrome on the more early stages) [24]. Further problem solving of the outlined issues will bring OND closer to standardized diagnostic technologies and to wider application in real medical practice.

\section{Acknowledgments}

This work was supported by Russian Foundation for Basic Research (grant №16-32-00662 mol_a) and partially by grant of the President of the Russian Federation for state support of young Russian scientists № MK-7168.2016.8. 